\title{
Il "desiderio" di Mattia Pascal ovvero Liolà : Pirandello maschilista?
}

Voler tornare ancora sul Fu Mattia Pascal potrebbe sembrare un'impresa azzardata. ${ }^{1}$ Vorrei comunque richiamare l'attenzione su un paradosso sottolineato da Vitilio Masiello ad un convegno dedicato esclusivamente a questo, il più noto $\mathrm{e}$ indubbiamente il più studiato dei romanzi pirandelliani:

A leggere [...] in controluce - o in sovraimpressione - il romanzo e le interpretazioni che se ne sono proposte, è difficile sottrarsi alla sensazione che la tradizione critica abbia in qualche modo contribuito piuttosto a ispessire che a diradare il tasso di ambiguità di un romanzo già di per sé inquietante e sfuggente; abbia ciò̀ contribuito ad accentuare l'originaria instabilità del sistema di significati di cui il testo è depositario, in termini che vanno ben al di là della costituzionale polisemia di un testo letterario in generale e di un testo pirandelliano in particolare. C'e, insomma, una sorta di "pirandellismo" - di situazione canonicamente "pirandelliana" - della vicenda critica: come se la natura ambigua, sfuggente, dell'opera e dell'autore irretisse i critici in un equivoco gioco di specchi, dove si confondono corpi e ombre, sostanze ed apparenze, verità ed inganno. (67)

Citare Masiello significa volersi allontanare in questo lavoro dal corto circuito dell' ambiguita in cui sembra essersi bruciata molta della critica, per puntare invece ad un'analisi precisa di un solo elemento: quello del desiderio. Complessità dunque, ma non ambiguità.

Tutti sono più o meno concordi nel ritenere Il fu Mattia Pascal uno dei primi esempi di anti-romanzo, di racconto cioè che illustra quello che Giancarlo Mazzacurati (190-97) ha chiamato "l'interdizione" o l'impossibilità del romanzo inteso in termini naturalistici e imperniato su una concezione del tempo "oggettivo" o storico, da Pirandello sentito come del tutto falso rispetto al tempo del "soggetto" e del suo desiderio. Ricordero che il romanzo fu scritto poco prima del saggio L'umorismo, la cui prima edizione (1908) reca la dedica "alla buon'anima di Mattia Pascal bibliotecario". Nel saggio Pirandello traccia la sua concezione dell'uomo e la visione dell'arte che ne deriva: l'uomo mentisce e l'arte (umoristica) smentisce. ${ }^{2}$ Narrato in prima persona, $l l$ Fu Mattia Pascal è quindi la testimonianza autobiografica di un uomo che, per il fatto stesso di essere uomo, o piuttosto "umano", non si conosce, e non può conoscersi. Il suo racconto ha valore, perciò, non come resoconto di un'esperienza vissuta ma come documento autonomo ed auto-referenziale, in cui tempo dell'azione e tempo della 
narrazione si fondono per creare un nuovo terzo tempo, ossia quello della temporalità bergsoniana della "durata". Le vicende umane si costruiscono attraverso una forte dimensione simbolica del grottesco, che tende a contrapporsi alle riflessioni di Mattia, rinvianti invece alla struttura profonda delle sue azioni e delle sue scelte "casuali". 3

Mattia attribuisce il fallimento della sua ricerca di una nuova identità all'impossibilità di esistere fuori dalle convenzioni sociali che regolano e danno forma alla nostra vita: nel caso suo, sarebbe l'impossibilità di acquistare una diversa identità anagrafica, e la conseguente impossibilità di dichiarare "onestamente" il suo amore ad Adriana, di denunciare il furto dei suoi soldi da parte del cognato di Adriana, ecc. Se tutti sono più o meno concordi nel ritenere che "l'inconsistenza anagrafica del personaggio" non è altro che "un ottimo alibi per giustificare la precipitosa fuga del nostro eroe" (Marchese 541), le diagnosi del male variano secondo l'orientamento del critico. Nel 1972 Roberto Alonge vedeva la storia di Mattia come "il romanzo dell'impossibilità del piccolo borghese di uscire dai limiti dal proprio ceto medio, di diventare altro, non piccolo borghese" (161). Arcangelo Leone De Castris vedeva più generosamente il fallimento di Mattia come la vittoria della "prigione delle forme convenzionali" al di fuori delle quali "c'è il nulla" (76-77). Secondo Giancarlo Mazzacurati, "lo impiglia e lo rigetta nel vuoto una sorta di neutralità impersonale degli apparati, un blocco che, prima ancora di manifestarsi come impossibilità burocratica, sembra già somatizzato da una passività di fondo incorporata nel personaggio, votato internamente alla resa, alla non-scelta" (216). Su posizioni diverse, Giuditta Rosowsky sottolinea che il problema va individuato, lacanianamente, nella famosa Loi du Père, ossia nella mancanza nella vita di Mattia della figura paterna con cui urtarsi e poi, infine, identificarsi. Mattia sarebbe, secondo la Rosowsky, "il figlio che non riesce a ri-conoscere la legge per il tangibile difettare dell'interdizione", e votato quindi a "appagare il desiderio materno e a costruirsi un'immagine di sé conforme a questo desiderio [. . . . . Il desiderio di produrre, di trasformare, non può affiorare, ché la madre si è imposta a lui come oggetto e come fine" (81-82); o, per dirla in termini più chiari ed accessibili con Angelo Marchese, "è innegabile in lui un complesso edipico che non gli consente un rapporto maturo con la donna" (541).

Recentemente, Romano Luperini e Giancarlo Mazzacurati hanno tentato di inquadrare il romanzo nell'ambito più vasto della "modernità" il primo, e del romanzo europeo che ne è il prodotto, il secondo. Ambedue gli interventi, estremamente suggestivi e corposi, sono imperniati sul concetto dell'allegoria (distinta dal simbolismo) come segno della modernità, della fine di ogni illusione di corrispondenza fra essere umano e natura, della "frattura fra livello sociale e livello naturale" (Luperini 211). Emblema di un'epoca in cui non vi è assolutamente più nessuna possibilità d'incontro fra i bisogni e le aspirazioni umane e ciò che l'assetto e le istituzioni sociali sono in grado di proporre, Mattia Pascal è, alla fine, "il personaggio che ha rinunciato ad essere persona" (Luperini 229) e 
l'opera "il primo romanzo politico del Novecento, la prima inchiesta sulla nuova figura di un soggetto senza riconoscibile società" (Mazzacurati 213). A livello della narrazione, questa frattura si riproduce in una specie di "allegoria rovesciata" (Mazzacurati 222), ossia "un gran paravento di rappresentazioni che dicono sempre altro da quello che lasciano vedere" (Mazzacurati 220).

Se tutto ciò è vero, e a mio parere lo è senz'altro, qual è il legame fra questa situazione e i "sintomi" di Mattia? Secondo Luperini, le spiegazioni offerte da Mattia per giustificare il suo comportamento e le sue scelte sono sempre così generiche e contraddittorie che "il lettore è indotto a cercare, sotto di esse, una motivazione rimasta inespressa" (231). Infatti, l'analisi di Luperini individua in modo esauriente, e davvero magistrale per la chiarezza, le manifestazioni della "malattia" di Mattia, ma senza andare oltre. È possibile farlo? C'è qualche cosa a livello del testo che ci autorizza a parlarne in termini più specifici di quanto non si è fatto finora? Donatella Stocchi-Perucchio osserva giustamente che il romanzo è una specie di giallo, fatto di "an overt surface and a mysterious substratum of loose hints" (7). Infatti, il discorso di Mattia è un miscuglio di "blindness and insight", in cui qualche volta Mattia, un po' come Edipo, "remains blind to the surface value of what he himself says"(82). Alla fine del romanzo, Don Eligio Pellegrinotto, il vecchio bibliotecario e interlocutore di Mattia, trae quelle che gli sembrano le conclusioni che si possono ricavare dalle avventure del collega: "Che fuori della legge e fuori di quelle particolarità, liete o tristi che sieno, per cui noi siamo noi, caro signor Pascal, non è possibile vivere" (249). Però, Mattia non è d'accordo: "Ma io gli faccio osservare che non sono affatto rientrato né nella legge, né nelle mie particolarità. Mia moglie è moglie di Pomino, e io non saprei dire ch'io mi sia" (250). Quindi non è vero - secondo Mattia — che non si possa vivere al di fuori delle convenzioni sociali, perché lo stanno facendo proprio lui, Pomino e Romilda. Quello che era "impossibile" a Roma a Miragno diventa perfettamente fattibile. Se a Roma lo stato civile ha trionfato (per dirla con Croce), a Miragno è stato nettamente sconfitto. Romilda ha due mariti - uno de iure, Mattia, e un altro de facto, Pomino. Ossia, come dice Mattia, egli e Pomino hanno la stessa moglie.

E se questo fosse il vero "desiderio" di Mattia? Di non lasciare mai Miragno, di vivere senza moglie, e di restare per sempre legato all'amico Pomino? Se invece di averlo semplicemente frustrato nelle sue aspirazioni, la sua "ombra" avesse trovato il modo di realizzarle a sua insaputa? Se il suo inconscio avesse sistemato le cose in modo tale da rispondere ai suoi bisogni più profondi, quelli stessi che egli non sa riconoscere, ma che determinano nel profondo le sue scelte, i suoi comportamenti, e il suo destino? Se, dicendo "mia moglie è moglie di Pomino", avesse dichiarato, inconsapevolmente quanto esplicitamente, di aver raggiunto il suo scopo?

Il motivo della donna condivisa più o meno tranquillamente da due uomini è abbastanza frequente nel repertorio pirandelliano. Basti pensare a Bellavita e al notaio Denora della novella L'ombra del rimorso (1914), a Carlino e a Tito della 
commedia $O$ di uno o di nessuno (1929), a Leone Gala e a Guido nel Giuoco delle parti (1919), a Giacomino e a Toti di Pensaci, Giacomino! (1916). In tutte queste opere, le donna in questione, invece di essere oggetto del desiderio di possesso esclusivo da parte degli uomini nella sua vita, serve per occultare e permettere la realizzazione indiretta di un altro desiderio, ossia il desiderio degli uomini di stabilire e mantenere un legame fra di loro. Le difficoltà sorgono quando una circostanza esterna viene a rompere l'equilibrio che si era creato e il rapporto tacito fra i due uomini entra in crisi: per esempio, per Carlino e Tito quando la donna si ritrova incinta e non si sa da chi, per Bellavita e il notaio Denora quando la moglie di Bellavita muore e il notaio non ha più motivo per venire a trovarlo nel suo caffe. La donna serve, per usare un termine di derivazione antropologica, come "oggetto di scambio", conduttore o mediatore di un rapporto in cui il vero "partner" è un altro uomo. ${ }^{4}$ Questo è particolarmente evidente nella novella $L$ 'ombra del rimorso, in cui, sulla scia della novella di Dostoevski L'eterno marito (1870), Pirandello si permette di fare a meno della donna completamente: la donna è morta, ed è sul rapporto fra il vedovo e l'amante che tutta la novella si concentra, senza "schermi" o mediazioni di sorta.

Ora, sia la prima parte del romanzo, in cui Mattia racconta le vicende della sua vita fino al momento della decisione di fingersi morto, sia l'ultima, in cui torna a Miragno dopo la sua "bislacca avventura" (249) per reinserirsi nella realtà di prima, s'iscrivono nel repertorio di questo motivo: non sono altro che il resoconto farsesco di una serie di "scambi" di donne fra uomini legati fra di loro da una complessa rete di rapporti di amore e odio, di amicizia e di rivalità, dissimulata da relazioni strumentali con donne, che gli permettono di inscenare i loro sentimenti intorno a dei pretesti socialmente accettabili. Infatti, le relazioni di Mattia con Romilda e Oliva non partono affatto da desideri spontanei ed autonomi, ma da desideri ispiratigli nel corso dei suoi rapporti con altri uomini. Mattia decide di fare la corte a Romilda quando viene a sapere che l'amico Pomino la desidera e che, per di più, la desidera anche l'odiato Batta Malagna, l'amministratore che ha approfittato della morte del padre di Mattia per sottrarre lentamente tutti i loro averi alla famiglia rimasta senza protezione. Mattia finge d'intromettersi per conto di Oliva, la moglie di Batta Malagna, ma in realtà è il desiderio di Pomino e di Malagna che ha fatto scattare il suo. L'ironia poi sta nel fatto che l'analisi del mimetismo di Pomino precede il paragrafo in cui Mattia riferisce la conversazione che darà l'avvio alla complicata tresca con Romilda e Oliva, senza mai riuscire a vedere il mimetismo nel proprio comportamento:

Mino era come l'ombra nostra; a turno, mia e di Berto; e cangiava con meravigliosa facoltà scimmiesca, secondo che praticava con Berto o con me. Quando s'appiccicava a Berto, diventava subito un damerino; e il padre allora, che aveva anche lui velleità, apriva un po' la bocca al sacchetto. Ma con Berto ci durava poco. Nel vedersi imitato finanche nel modo di camminare, mio fratello perdeva subito la pazienza, forse per paura del ridicolo, e lo bistrattava fino a cavarselo di torno. Mino allora tornava ad appiccicarsi a me; e il padre a stringer la bocca al 
sacchetto. ...

Ora Mino, un giorno, a caccia, a proposito del Malagna, di cui gli avevo raccontato le prodezze con la moglie, mi disse che aveva adocchiato una ragazza, figlia d'una cugina del Malagna appunto, per la quale avrebbe commesso volentieri qualche grossa bestialita. Ne era capace; tanto più che la ragazza non pareva restía; ma egli non aveva avuto modo finora neppure di parlarle[... . .

[. . .] volli tentare, per il bene d' Oliva, se mi fosse riuscito d'appurare qualche cosa [... . . Mino mi si raccomandò per la ragazza.

"Non dubitare", gli risposi. "La lascio a te, che diamine!" (25-26)

L'ironia di Pirandello è particolarmente feroce quando Mattia, facendosi il paladino degli interessi di Oliva, Pomino e Romilda, dice di voler salvare Romilda da "un mercato infame": se è vero che la madre di Romilda sta cercando di "vendere" la figlia al Malagna, è altrettanto vero che il "mercato", nel senso di "scambio di merci", sarà quello messo in moto dai desideri confusi di Mattia. Egli stesso, del resto, riconosce di non esser stato mosso da "amore" nei confronti di Romilda, ma non sa dare un nome ai suoi desideri:

Perché mostravo tanta smania di maritar Romilda? Per niente: ripeto: per il gusto di stordire Pomino [...] .

Che colpa ho io se Pomino eseguì con troppa timidezza le mie prescrizioni? che colpa ho io se Romilda, invece d'innamorarsi di Pomino, s'innamorò di me, che pur le parlavo sempre di lui? [...] .

[...]. Mi ero poi innamorato anch'io di Romilda, pur seguitando sempre a parlarle dell'amore di Pomino; innamorato come un matto di quegli occhi belli, di quel nasino, di quella bocca, di tutto, finanche d'un piccolo porro ch'ella aveva sulla nuca, ma finanche d'una cicatrice quasi invisibile in una mano, che le baciavo e le baciavo e le baciavo ... per conto di Pomino, perdutamente. (30-31)

Senonché Mattia si troverà ad essere egli stesso oggetto di un inganno, quello ordito dalla madre di Romilda ai danni di lui. Appena viene a sapere che sua figlia è incinta, la costringe - o almeno così dirà Romilda - a lasciare Mattia e a convincere Malagna che il figlio è suo e che perciò non è colpa di lui se sua moglie, Oliva, non riesce ad aver figli (come invece lo è). Quando Oliva viene a sapere che il marito la vuole lasciare per Romilda si lascia convincere da Mattia che anche lei deve fare in modo da trovarsi incinta:

"E dunque?" dissi io, ridendo acre. "Vedi? Tu non puoi più ottenere nulla negando. Te ne devi guardar bene! Devi anzi dirgli di sì, che è vero, verissimo ch'egli può aver figliuoli ... comprendi?" (33)

E così, spinto a detto di lui "dal pensiero della povera Oliva [. . . ] e dalla speranza di fare un bene a quella ragazza che veramente [gli] aveva fatto una grande impressione" (30), Mattia finisce per per "vendicarsi" due volte del Malagna, rendendo incinte due donne. Alla fine il Malagna rinuncia a Romilda, come previsto da Mattia, ma impone a Mattia delle nozze riparatrici con lei, accettando di man- 
tenere il figlio viste le tristi condizioni finanziarie del suo sostituto. A questo punto Mattia (il fu) fa un commento in cui rivela molto chiaramente, sia pure sotto il velo dell'ironia, quale avrebbe potuto essere il suo desiderio in tutto questo:

e concluse che egli non avrebbe potuto infine far altro che provvedere - a patto però che si fosse serbato con tutti il massimo segreto - provvedere al nascituro, fargli da padre, ecco, giacché egli non aveva figliuoli e ne desiderava tanto e da tanto tempo uno.

Si può essere - domando io - più onesti di così?

Ecco qua: tutto quello che aveva rubato al padre egli lo avrebbe rimesso al figlio nascituro. (35)

Dopo il matrimonio, che molto presto si rivela essere stato uno sbaglio, Mattia incontra per la strada Pomino, che cerca di evitarlo:

Me ne voleva [...] me ne voleva, Pomino, del tradimento che, a suo credere, gli avevo fatto [...] .

Mi venne allora la tentazione di domandargli perché dunque, se veramente egli n'era così addogliato, non l'aveva sposata lui, Romilda, a tempo, magari prendendo il volo con lei, com'io gli avevo consigliato, prima che, per la sua ridicola timidezza o per la sua indecisione, fosse capitata a me la disgrazia d'innamorarmene; e altro, ben altro avrei voluto dirgli, nell'orgasmo in cui mi trovavo; ma mi trattenni. Gli domandai, invece, con chi se la facesse, di quei giorni. "Con nessuno!" sospirò egli allora. "Con nessuno! Mi annojo, mi annojo mortalmente!"

Dall'esasperazione con cui proferì queste parole mi parve d'intendere a un tratto la vera ragione per cui Pomino era così addogliato. Ecco qua: non tanto Romilda egli forse rimpiangeva, quanto la compagnia che gli era venuta a mancare; Berto non c'era più; con me non poteva più praticare, perché c'era Romilda di mezzo, e che restava più dunque da fare al povero Pomino? (43-44)

Alla fine del romanzo, fallito il tentativo di rifarsi una vita come Adriano Meis, Mattia torna a Miragno. Qui, di nuovo, sarà questione del rapporto con Romilda, perché durante la sua assenza Pomino se l'è sposata ed hanno avuto una figlia insieme. La scena in cui Pirandello rappresenta l'incontro dei tre è chiaramente una scena in cui i due maschi sono i protagonisti, e Romilda una figurante, conduttrice del rapporto di amore-odio fra i due vecchi amici. Del resto, Mattia ammette apertamente che non ha nessun sentimento nei confronti di Romilda, e che essa lo interessa in quanto bella e in quanto gli avrebbe permesso di vendicarsi di Pomino, o riprendendola o non riprendendola, a seconda dei sentimenti di Pomino. È da notare, inoltre, che il desiderio eventuale di Romilda non figura assolutamente nel ragionamento di Mattia:

Romilda, indossata la veste da camera, venne a raggiungerci. Io rimasi a guardarla alla luce, ammirato: era ridivenuta bella come un tempo, anzi più formosa.

"Fammiti vedere. .." le dissi. "Permetti, Pomino? Non c'è niente di male: sono marito anch'io, anzi prima e più di te. Non ti vergognare, via, Romilda! Guarda, 
guarda come si torce Mino! Ma che ti posso fare se non son morto davvero?"

"S'inquieta!" feci, ammiccando, a Romilda [...] .

Mi accostai a Romilda e le scoccai un bel bacione su la guancia.

"Mattia!" gridò Pomino, fremente.

Scoppiai a ridere di nuovo.

"Geloso? di me? Va' là! Ho il diritto della precedenza. Del resto, su, Romilda, cancella cancella [. . .] . Guarda, venendo, supponevo (scusami, sai, Romilda), supponevo, caro Mino, che t'avrei fatto un gran piacere, a liberartene, e ti confesso che questo pensiero m'affligeva moltissimo, perché volevo vendicarmi, e vorrei ancora, non credere, togliendoti adesso Romilda, adesso che vedo che le vuoi bene e che lei ... sì, mi pare un sogno, mi pare quella di tant'anni fa ... . (241-42)

L'ultimo "scambio di donna", però, non ha luogo; Mattia dice che non vuole riprendere Romilda se ciò significa "lasciare una figliuola senza mamma" (240); ma perché credere che questa sia la vera ragione, se si è sempre attribuito motivi tanto altruistici quanto illusori? A pensarci bene, invece, questo gli permette di consolidare il legame con Pomino: un legame a cui Pirandello gli fa accennare velatamente, confondendo un po' le acque: "Vedi, Romilda? Abbiamo fatto pari e patta: io ho un figlio, che è figlio di Malagna, e tu ormai hai una figlia, che è figlia di Pomino" (ibidem). In realtà, Mattia e Malagna hanno lo stesso figlio, mentre Mattia e Pomino hanno la stessa moglie: ormai Mattia è legato inestricabilmente ai due uomini che sono sempre stati i veri oggetti dei suoi investimenti emotivi. Se si riprendesse Romilda, il legame di Romilda con Pomino si dissolverebbe, mentre il suo legame con Romilda non può dissolversi perché, nonstante la sua morte anagrafica, secondo la legge è ancora lui il "vero" marito di Romilda. Il legame con Fomino, invece, dipende dalla possibilità di mantenere il legame di Romilda con Pomino che, visto che non esiste de iure, deve esistere per forza de facto. La cosa è spiegata in termini minuziosi a Pomino dallo stesso Mattia, il quale però si dimostra del tutto ignaro del suo significato recondito:

"Ma il matrimonio s'annulla?" gridò Pomino.

"E tu lascialo annullare!" gli dissi. "Si annullerà pro forma, se mai: non farò valere i miei diritti e non mi farò neppure riconoscer vivo ufficialmente, se proprio non mi costringono. Mi basta che tutti mi rivedano e mi risappiano vivo di fatto [. . .] il resto non m'importa! Tu hai contratto pubblicamente il matrimonio; è noto a tutti che lei è, da un anno, tua moglie, e tale rimarrà. Chi vuoi che si curi più del valor legale del suo primo matrimonio? Acqua passata ... Romilda fu mia moglie: ora, da un anno, è tua, madre d'una tua bambina. Dopo un mese non se ne parlerà più. Dico bene, doppia suocera?" (242)

Il rapporto fra Mattia e Pomino è stato variamente commentato dalla critica: Marchese fa notare che "Mattia è attratto dal ruolo di mediatore" e che "ha un suo doppio non tanto nel fratello Berto quanto in Gerolamo Pomino" (544); la Stocchi-Perucchio rileva che Pomino è dotato di una "'meravigliosa facoltà scimmiesca', by virtue of which he could become a sosia of either one of the two broth- 
ers" (28); Luperini vede giustamente il rapporto di Mattia con Pomino come il primo di una serie, osservando che Mattia ha una "irresistibile vocazione [a] inserirsi come terzo in una situazione di coppia (uomo-donna) già esistente [. . .] con un attegiamento di rivalità nei confronti di una figura paterna" determinato da una "coazione [a ripetere] che lo induce alla trasgressione ma non a rovesciare il rivale" (232-33). Ma è forse Lucio Lugnani, nel suo bel saggio L'infanzia felice che mette il dito più direttamente sulla piaga, anche senza fare riferimento esplicito al Fu Mattia Pascal :

Negli amici-gemelli i protagonisti pirandelliani cercheranno in fondo sempre la replica, e il sostitutivo, di quella felice condizione perduta di complementaritàidentità, cercheranno l'altra metà di Narciso, ossia l'immagine materna, e la pacifica omosessualita infantile. Ma l'ombra della madre e del rapporto di perfetta identità-compenetrazione con lei segnerà per sempre come imperfetto il rapporto con l'altro; soprattutto l'eterosessualità della relazione fra amanti e fra coniugi, fra uomo e donna non potrà perciò mai essere felice [. . . ] e nasconderà sempre una certa dose di odio e di ribrezzo per l'altro, per colui che ha preso il posto della madre. Il rapporto eterosessuale contiene sempre qualcosa della violenza traumatica connessa alla pubertà e alla morte della madre, e perciò numerosi personaggi tenderanno per vie diverse alla omosessualità latente del rapporto gemellare come all'unica possibile, per quanto inconscia, riproduzione nel mondo adulto della felice condizione infantile. (116)

E molto rivelatrice, se letta dal punto di vista delle indicazioni di Lugnani, la descrizione che Mattia/Adriano ci fa dei suoi sentimenti nei confronti di Adriana, la donna di cui si considera innamorato (del resto, la scelta del nome "Adriana" da parte di Pirandello per questo personaggio, quando Mattia si è battezzato "Adriano", la dice più lunga di qualunque analisi su ciò che Pirandello ha voluto dire):

Le anime hanno un loro particolar modo d'intendersi, d'entrare in intimità, fino a darsi del tu, mentre le nostre persone sono tuttavia impacciate nel commercio delle parole comuni, nella schiavitù delle esigenze sociali. Han bisogni lor proprii e loro proprie aspirazioni le anime, di cui il corpo non si dà per inteso, quando veda l'impossibilità di soddisfarli e di tradurle in atto. E ogni qualvolta due che comunichino fra loro così, con le anime soltanto, si trovano soli in qualche luogo, provano un turbamento angoscioso e quasi una repulsione violente d'ogni minimo contatto materiale, una sofferenza che li allontana e che cessa subito, non appena un terzo intervenga. Allora, passata l'angoscia, le due anime sollevate si ricercano e tornano a sorridersi da lontano.

Quante volte non ne feci l'esperienza con Adriana! Ma l'impaccio ch'ella provava era allora per me effetto del natural ritegno e della timidezza della sua indole, e il mio credevo derivasse dal rimorso che la finzione mi cagionava, la finzione del mio essere, continua, a cui ero obbligato, di fronte al candore e alla ingenuità di quella dolce e mite creatura. (156-57)

Lugnani vede nella "cognizione drammaticamente acquisita delle dicotomie 
io/altro e dentro/fuori" la fonte dell'incapacità di affrontare la vita adulta: infatti, nel brano seguente, l'uso della metafora della finestra da parte di Mattia nel parlare di Adriana indica come correre il rischio della relazione significhi, per lui, "guardare fuori":

Sì, forse anch'ella istintivamente obbediva al bisogno mio stesso, al bisogno di farsi l'illusione d'una nuova vita, senza voler saper né quale né come. Un desiderio vago, come un'aura dell'anima, aveva schiuso pian piano per lei, come per me, una finestra nell'avvenire, donde un raggio dal tepore inebriante veniva a noi, che non sapevamo intanto appressarci a quella finestra né per rinchiuderla né per vedere che cosa ci fosse di là. (157)

Da questo stesso trauma muoveranno, secondo Lugnani, "le mediazioni sempre difficili fra una sede naturale e natale e un luogo lontano che sradica e spaesa colui che attraverso il viaggio (l'altro grande mediatore) vi perviene e che da esso agogna ritornare" (125). Letta in questa ottica, non appare affatto casuale la descrizione che Mattia fa della sua vita dopo il ritomo a Miragno:

Basta. Io ora vivo in pace, insieme con la mia vecchia zia Scolastica, che mi ha voluto offrir ricetto in casa sua. La mia bislacca avventura $m$ 'ha rialzato d'un tratto nella stima di lei. Dormo nello stesso letto in cui morì la povera mamma mia, e passo gran parte del giorno qua, in biblioteca, in compagnia di Don Eligio, che è ancora ben lontano dal dare assetto e ordine ai vecchi libri polverosi. (249)

Si ricorderà che la zia Scolastica è la sorella zitellona della madre che non ha voluto mai sposarsi "non già perché non si fosse sentita disposta ad amare, ma perché il piu lontano sospetto che l'uomo da lei amato avesse potuto anche col solo pensiero tradirla, le avrebbe fatto commettere - diceva - un delitto":

Tutti finti, per lei, gli uomini, birbanti e traditori. Anche Pomino [padre]? no, ecco: Pomino, no [...]. . Di tutti gli uomini che avevano chiesto la sua mano, e che poi si erano ammogliati, ella era riuscita a scoprire qualche tradimento, e ne aveva ferocemente goduto. Solo di Pomino niente; anzi il pover'uomo era stato un martire della moglie.

E perché dunque, ora, non lo sposava lei? Oh bella, perché era vedovo! Era appartenuto a un'altra donna, alla quale forse, qualche volta, avrebbe potuto pensare. (12)

Anche la zia Scolastica dunque è incapace di pensare il suo desiderio se non in presenza di un terzo: è afflitta da una forma di gelosia simile a quella di Rico Verri in Leonora, Addio! (1910) e in Questa sera si recita a soggetto (1930). Rico Verri sposa Momina, che egli sa aver avuto un passato che lo tormenterà, proprio per poterla tener poi sotto chiave e dare così un contenuto alla sua gelosia congenita. La zia Scolastica, in quanto donna, semplicemente non si sposa. Il fatto che Pirandello faccia finire Mattia con lei non credo sia senza significato, come non lo è il fatto che lo faccia finire anche nel letto della madre - proprio come Pepè Alletto, il protagonista inetto de Il turno (1895), il quale non se n'era mai completamente staccato. Un altro particolare significativo si ha, credo, 
all'inizio, nella Premessa seconda (filosofica) a mo' di scusa, in cui Mattia descrive la confusione nella vecchia biblioteca, tale che "si sono strette per la vicinanza fra questi libri amicizie oltre ogni dire speciose":

don Eligio Pellegrinotto mi ha detto, ad esempio, che ha stentato non poco a staccare da un trattato molto licenzioso Dell'arte di amar le donne, libri tre di Anton Murzio Porro, dell'anno 1571, una Vita e morte di Faustino Materucci, Benedettino di Polirone, che taluni chiamavano beato, biografia edita a Mantova nel 1625 . Per 1'umidità, le legature de' due volumi si erano fraternamente appiccicate. Notare che nel libro secondo di quel trattato licenzioso si discorre a lungo della vita e delle avventure monacali. (6)

Questo accostamento provocatorio non esprime in fondo l'essenza della vita degli uomini su cui Pirandello punta la sua lente deformante in questo romanzo? Le donne possono avere funzione erotica, ma i veri compagni, i veri oggetti di amore - e di odio - sono altri uomini.

\section{Liolà}

In un interessante saggio pirandelliano intitolato "Madri, puttane, schiave sessuali e uomini soli" Roberto Alonge osserva giustamente che nell'universo pirandelliano, "solo gli uomini, solo i maschi riescono a comunicare ancora tra di loro", tant'è vero che "l'eroe pirandelliano nasce sempre in questa condizione ambientale, mentre parla ad un altro uomo"(91). Anche il racconto di Mattia è un racconto fatto ad un altro uomo, il vecchio bibliotecario Don Eligio Pellegrinotto, con cui Mattia divide le sue giomate di lavoro. Alonge non parla di Mattia direttamente, ma vede in Liolà, protagonista della famosa commedia campestre tratta da Pirandello dai capitoli tre e quattro del Fu Mattia Pascal, "l'immagine indimenticabile del prototipo dell'uomo solo" il quale "nel suo apparire episodico ma ricorrente lungo l'intero arco della produzione drammaturgica, è tale perché ha consumato un rifiuto radicale e perenne della donna" (24). Infatti, la mia ipotesi è che Pirandello considerasse la situazione descritta in questi capitoli in qualche modo "archetipica", e perciò decise di inscenarla su uno sfondo pastorale, ossia "vicino alla natura" e fuori del tempo. Inoltre, Liolà si svolge all'insegna di un'allegria tutt' altro che tipicamente pirandelliana, almeno per quanto riguarda il protagonista: a differenza di Mattia narratore, Liolà è un personaggio spensierato, proprio come lo era Mattia personaggio prima di essere stato costretto ad affrontare la vita adulta tramite il matrimonio. Si noti però che nella commedia la trasposizione comprende unicamente la dinamica del rapporto fra Mattia e Batta Malagna, usurpatore della sostanza della sua famiglia - manca completamente un personaggio il cui ruolo corrisponda a quello dell'amico Pomino. Su questo si tornerà in seguito.

In Liolà il ruolo di Batta Malagna, cioè del personaggio sterile, è attribuito a zio Simone, un ricco contadino che ha sposato la giovane Mita (Oliva) nell'unica speranza di aver un figlio. Mita però non ingravida, per ragioni ovvie, e Simone 
la picchia e la maltratta come se la colpa fosse senz'altro, e unicamente, di lei. Liolà è un semplice bracciante che non ha i soldi di Simone, ma in compenso riesce a far fruttare ogni donna con cui giace, tant'è vero che ha tre figli da tre donne diverse, allevati da sua madre, senza che egli ne abbia sposato nessuna. Il tesoro di Liolà è il suo seme, quello di Simone la sua roba. Tuzza (Romilda), nipote di Simone, decide di approfittar di ambedue, facendosi ingravidare dal giovane ed aitante Liolà, e poi mettendosi d'accordo con lo zio Simone per fingere che il figlio è suo e così farne l'erede della sua sostanza. Quando Liolà comincia a sospettare di essere stato strumentalizzato, chiede la mano di Tuzza per impedire l'infame mercato. Tuzza, ovviamente, non ne vuole sapere e allora, per sventare il suo piano, Liolà ingravida anche Mita, moglie di Simone, e Tuzza si trova scartata da Simone a favore della moglie. Tuzza, per la rabbia, cerca di accoltellare Liolà, ma egli la disarma e la commedia si chiude su un tono "allegro", con Liolà che ora rifiuta di sposare Tuzza, ma accetta di far allevare anche questo quarto figlio dalla madre.

Abbiamo detto che manca completamento il personaggio che fa le veci di Pomino - manca cioè il "doppio", l'amico-gemello in cui il protagonista pirandelliano cerca, per dirla con Lugnani "in fondo sempre la replica, e il sostitutivo, di quella felice condizione perduta di complementarità-identità [. . . ] l'altra metà di Narciso, ossia l'immagine materna, e la pacifica omosessualità infantile". La ragione per cui manca è semplice, e spiega anche l'insolita "allegria" della commedia, che lo stesso Pirandello stentava a capire. ${ }^{5}$ Liolà non ha bisogno di "sostitutivi". Vive in una specie di paradiso terrestre in cui gli è consentito vivere ed affermare la sua "virilità" senza per questo dover lasciare la madre e tutto ciò che ella simbolizza. Mattia è stato costretto a sposare Romilda, mentre Liolà vive al di fuori delle convenzioni sociali. Tutti i figli di Liolà, avuti da donne diverse che vengono regolarmente scartate non appena nato il bambino, sono allevati da sua madre, alla quale egli rimane sempre legato: onde la sua felice spensieratezza. La "moglie" di Liolà è sua madre, le altre donne sono semplicemente contenitori del suo seme. Liolà può vivere la sua sessualità senza dover riconoscere la radicale alterità della donna come soggetto, senza dover rinunciare all'illusione narcisistica infantile, senza dover patire la divisione dell'io che segue alla rinuncia alla madre come oggetto. Liolà realizza il "desiderio" di Mattia senza pagare lo scotto sociale esatto da Mattia - l'emarginazione, e per un mero "sostitutivo". Mattia è Liolà nevrotizzato, imborghesito, civilizzato, represso. Invece Liolà è a contatto con tutti i suoi bisogni e desideri più istintivi e pud̀ soddisfarli senza problemi. Roberto Alonge dice addirittura che

Liold è il preludio negato dell'intera drammaturgia pirandelliana [ . . ] la reverie delirante e inconfessata del grande maschio solitario [. . . l'utopia di una societa senza madre, in cui i figli nascano direttamente dal grembo dei padri, o al massimo, in cui la donna sia soltanto il ricettacolo temporaneo - per i nove mesi indispensabili - del seme dell'uomo. (94) 
Dove non mi posso trovare d'accordo con Alonge è nelle sue conclusioni:

Dobbiamo riconoscere in Pirandello [. . . ] le linee di una grande, reazionaria fantasia, patriarcale e maschilista. Ma è proprio questa, mi pare, la sua attualità. Pirandello ci aiuta a vedere in noi stessi. Perché è tempo, per noi maschi, di cominciare a guardarci per quello che siamo. E non per quello che, forse, vorremmo essere. (109-10)

Alonge sembra voler far coincidere il punto di vista dei maschi pirandelliani con l'autore stesso. Io, invece, farei valere il principio secondo cui l'autore implicito di un testo non collima mai con i suoi personaggi: nell'opera pirandelliana non ci sono "le linee di una grande fantasia patriarcale e maschilista" ma direi piuttosto il ritratto analitico, acuto e spietato, di una società patriarcale e maschilista. E ciò si vede molto chiaramente proprio in Liolà, in cui l'ambiente "primitivo" (fra "zotici", come dice lo stesso protagonista) permette ai personaggi di chiamare le cose col loro nome piu facilmente che non sia il caso nelle opere di ambientazione borghese.

"Ma è forse vergogna per un uomo non aver figliuoli?" chiede "con aria ingenua" Ciuzza. E semplicemente "segno che Dio non ha voluto dargliene", dice Nela, seguita da Luzza che chiede, logicamente, "perché allora se la piglia con la moglie?" Tutte e tre son fatte subito tacere da Zia Croce - "Zitta tu! Questi non son discorsi in cui possano metter bocca le ragazze" (atto I) la quale si attacca invece al motivo più convezionale dell'erede per spiegare la disperazione dello zio Simone: "No, piange - siamo giuste - piange per la roba; tanta bella roba che, alla sua morte, andrebbe a finire in mano d'altri. Non se ne sa dar pace!" (atto I). Intanto, da queste battute s'è capito che la posta in gioco è la virilità dello zio Croce, e non la sua roba. Infatti Liolà, a cui manca la roba ma abbondano i figli, fa chiaramente sentire allo zio Simone che, se è vero che la roba conta, ciò che la roba non può comprare è proprio il figlio, perché il figlio, come la donna, è un oggetto e uno strumento: è simbolo della virilità del suo "proprietario", di chi lo ha generato, e di nessun altro:

Hanno messo una legge nuova, fatta apposta per noi. Dico, per alleggerire le nostre popolazioni. Stia a sentire. Chi ha una troja che gli fa venti porcellini, è ricco, non è vero? Se li vende; e più porcellini gli fa, più ricco è. E così una vacca; quanti più vitellini gli fa. Consideri ora un pover'uomo con queste donne nostre che Dio liberi, appena le tocca, patiscono subito di stomaco. È una rovina, no? Bene, il Governo ci ha pensato. Ha messo la legge che i figli, d'ora in poi, si possono vendere. Si possono vendere e comprare, zio Simone. E io, guardi, (gli mostra $i$ tre bambini) posso aprir bottega. Vuole un figlio? Glielo vendo io. Qua, questo". (Atto I)

Il fatto che alla fine Tuzza tenti di accoltellarlo per la rabbia, e che Mita sia ora condannata a restare con un uomo che la maltratta, è minimizzato da Liola con la sua bonaria allegria da stallone errante ("pronto ha ghermito il braccio di 
Tuzza, e con l'altra mano le batte sopra le dita fino a farle cadere il coltello a terra, ride e rassicura tutti, che non è stato nulla" [atto III]) ma non per questo va cancellato dal novero degli effetti sprigionati dallo scambio di posizioni che egli esegue così abilmente a scapito di tutti. È vero che Tuzza ha voluto servirsi di lui, ma per acquistare quell'"oggetto di valore" - il figlio — che le avrebbe permesso di avere un uomo. È da notare che Zia Croce, in quanto cugina di Simone, avrebbe comunque ereditato da lui, in quanto, secondo la legge, se non ci sono figli, il patrimonio va alla parentela tutta. Quindi Tuzza, figlia di Zia Croce, non vuole tanto la ricchezza di Simone, quanto l'uomo in sé. Come spiega alla madre, è gelosa di Mita, perché Mita, prima di sposare Simone era stata amata da Liolà che, poi, ha continuato ad andarle appresso nonostante che fosse sposata. Mita aveva marito e amante, mentre lei, Tuzza, non aveva né l'uno né l'altro: "Quante cose doveva avere quella morta di fame? Non bastava il marito ricco? Anche l'amante festoso?" (Atto I).

Mita intanto, è maltrattata e percossa così tanto che sua zia Gesa, che aveva creduto di far bene a suo tempo, ora rimpiange la decisione di dare la nipote orfana a Zio Croce: "Vorrei vedere se avesse almeno un fratello! Non la tratterebbe così, ve l'assicuro io! Per miracolo non se la pesta sotto i piedi: avete veduto!" (atto I). Il finale non è, quindi, così allegro come sembra, e celebra non tanto la virilità spensierata di Liolà, quanto la sua soverchieria mascherata da cavalleria nei confronti di Mita. Dice di averla voluta aiutare a conservarsi il marito quando lei, ormai, ne avrebbe fatto volentieri a meno: "Gli altri t'insegnano come si fa, e tu non vuoi seguir l'insegnamento. Gliela lasci commettere tu a Tuzza l'infamia, non io! Perciò io ho negato e nego! Per te, per te nego, per il tuo bene, e perché non c'è altro mezzo ora di sventare quest'inganno e quest'infamia!" (atto II). La realtà è che Liolà vuole regolare i suoi conti con Simone, costi quel che costi, e non importa a chi, basta che non sia a lui. E infatti, alla fine, la roba di Simone sarà ereditata da suo figlio, anche se non porterà il suo nome. Tuzza, la "furba gelosa", intanto rimarrà senza uomo e senza figlio, perché il bambino le sarà "espropriato", per essere allevato, come gli altri, dalla madre di Liolà. Le vere perdenti, in questo gioco, sono le donne, sia che rimangano con uomini, sia che ne rimangano senza. Liolà fa volentieri a meno della moglie, tanto ciò che conta sono i figli, e Zio Simone è disposto a spacciare per suo il figlio di chiunque, pur di averlo; le donne, con l'eccezione di Mita, che non ci crede più ma a cui comunque è stato tolto il potere di decisione, non riescono a pensarsi se non in relazione all'uomo: la loro soggettività al di fuori della loro funzione nel sistema patriarcale non esiste. Ė ciò che fa notare Zia Ninfa esplicitamente, sotto il solito velo dell'allegria, quando fa il verso alle donne che si facevano vento coi ventagli alla messa delle signore:

Le signorine da marito così: "L'avrò! l'avrò! l'avrò!" Le signore maritate così: "Io ce l'ho! io ce l'ho! io ce l'ho! Mentre le povere vedove: "L'avevo e non l'ho più! l'avevo e non l'ho più! l'avevo e non l'ho più!" (Atto I) 


\section{Feminismo e Pari}

Mi sembrerebbe eccessivo proporre un'analisi in cui Pirandello appare femminista - non credo certo che potesse essere realmente a favore di cio che le femministe del suo tempo rivendicavano. Penso invece che considerasse la società in cui viveva retta sulla negazione e la paura della donna: da ciò la sua oggettivazione e strumentalizzazione.

Bisogna tener presente che Pirandello costruisce la psicologia dei suoi personaggi in termini sociali, generalizzabili a tutta una cultura e/o a tutta una generazione. ${ }^{6}$ In questo senso, il progetto pirandelliano continua e allo stesso tempo va oltre quello naturalistico. Il naturalismo studia l'evoluzione dell'individuo nel suo contesto sociale, in base ad un modello di causa ed effetto, ma parte sempre dall'individuo inteso in termini romantici: un nucleo "originario" su cui poi vengono ad imprimersi una serie di influssi sociali esteriori, non sempre uguali. Il personaggio pirandelliano invece non è ostacolato ma creato dal sociale, ossia segnato dalla posizione che occupa nel sistema: antropologicamente, la sua struttura psichica è il risultato dell'interiorizzazione di un insieme di relazioni sociali. (Il meccanismo di causa ed effetto non è abbandonato, è semplicemente applicato al rapporto fra un'ipotetica "psiche sociale" del personaggio e le sue esperienze, e rappresentato come si manifesta spesso nella vita, senza nessi logici apparenti.) Le esperienze dei personaggi sono sempre emblematiche, tipiche e non individuali. ${ }^{7}$ Le loro frustrazioni rinviano non, romanticamente, a desideri "autentici" ma irrealizibili, ma a "i fantasmi interiori e inconsci di un io diviso", condizione inderogabile del loro inserimento nel sistema. Le insoddisfazioni sono parte integrante della "maschera" ossia della "parte": perciò non hanno soluzioni. Il copione è stato scritto altrove, ed è sempre lo stesso. Perciò, nei Sei personaggi, i personaggi sono Il Padre, La Madre, Il Figlio, La Figliastra, tout court: rappresentano delle funzioni "eterne". Così anche Mattia non rappresenta una "persona", ma un ruolo, come i sei personaggi. Cið che di Mattia interessa a Pirandello sono le caratteristiche psichiche fisse che costituiscono la struttura profonda ed immutabile del suo comportamento, e del suo modo di costruire il mondo, e che dipendono dalla sua "posizione" nel sistema.

Il comportamento di Mattia, come quello di Liolà del resto, denuncia sostanzialmente, come è stato notato da altri, la mancanza di un modello maschile adeguato. Questo è confermato dall'assenza quasi totale di figure paterne positive nel corpus pirandelliano, quando non della loro assenza tout court. ${ }^{9}$ Se la figura paterna è il tramite fra mondo infantile e realtà sociale - se è cioè attraverso il padre che l'essere umano s'iscrive nel "sociale" - , allora ciò che Pirandello sta effettivamente descrivendo è una società senza "padri' in grado di iniziare i figli ad una mascolinità "vera". Ciò che Mattia non sa fare è essere "uomo", nel senso borghese, ottocentesco del termine. Non sa essere produttivo e procreativo, lavorare e mettere su famiglia, assumere il ruolo sociale del maschio. Non sa neanche essere protettivo. Non sa difendere il patrimonio familiare né la madre contro i 
soprusi del Malagna e della vedova Pescatore, né sa difendere i suoi soldi e Adriana contro Papiano, nonostante l'ostentata - a parole - premura in proposito. La crisi dei valori di cui tanto si è parlato in rapporto agli anni fra il 1880 e il 1920 circa, si manifesta in Pirandello soprattutto, credo, come crisi della mascolinità, che consiste ormai quasi unicamente nella difesa dei propri "diritti naturali" nei confronti della donna, e del proprio "prestigio" nei confronti di altri maschi, in ambedue i casi a costo della strumentalizzazione e della riduzione a oggetto della donna. Il maschio si riconosce solo attraverso la prepotenza o la passività, due facce della stessa medaglia.

La complessità della visione pirandelliana si può ricavare, oltre che dalle grandi opere, ${ }^{10}$ dal confronto di due suoi scritti sull'argomento, un articolo giornalistico intitolato Feminismo (1909), e un racconto espressionistico intitolato Pari (1907). Il racconto è utile ai nostri fini sia perché costituisce un approfondimento fantastico del punto di vista espresso nell'articolo, sia perché in esso Pirandello collega esplicitamente la problematica dell'"uomo solo", come lo chiama Alonge, all'antifemminismo, fatto coincidere sostanzialmente con la paura delle donne.

"Feminismo", pubblicato su La preparazione del 27-28 febbraio 1909,11 è un articolo estremamente ambiguo. L'ambiguità è dovuto al fatto che contiene due, anzi tre voci - quella del narratore anonimo, quella del suo interlocutore, certo dottor Paulo Post, e quella della figlia del dottore, la femminista Pietra, dalla voce "gutturale, maschile" e dalla "testa rossa, arruffata, con gli occhi a staffa, che le ingrandivano enormemente e confusamente gli occhi". Il colloquio ha luogo nello studio del dottore, ove lavora silenziosamente in fondo anche la figlia, dietro una "mastodontica scrivania sovraccarica anch'essa tutt'intorno di libri e di carta ammucchiata". Il dottor Post ha l'abitudine di sottoporre le cose al famoso "cannochiale rivoltato" pirandelliano, e cioè di vederle "da lontano", e perciò viene interrogato dal narratore intorno alle "più vive questioni sociali del momento". Il 'feminismo' però viene affrontato dal dottore "con una certa inquietudine" per via della presenza della figlia, la quale invece incoraggia il padre ad esprimersi apertamente, senza badare a lei: "Parla pure, papà — disse quella voce" (1068-69).

Secondo il dottore "il femminismo è, al pari di tante e tant'altre cose, una costruzione ideale dei nostri giorni" (1070), espressione della frustrazione sessuale delle donne, che hanno sempre più difficoltà a trovar marito perché gli uomini a loro volta hanno sempre più difficoltà a guadagnarsi la vita e a mantenere decorosamente una famiglia:

Il veder frustata, intanto, la loro naturale inclinazione [. . . ] il dover soffocare il loro bisogno istintivo, le ha un po' esasperate e le fa un po' farneticare . . . Tutte queste prediche fervorose per la così detta emancipazione della donna, che altro sono in fondo se non una sdegnosa mascherata del bisogno fisiologico, che si muove sotto? (1071) 
L'articolo si conclude con un'ultima tirata del dottore, seguita da un vano tentativo del narratore anonimo di sentire anche il parere della figlia:

Supporre che la donna, praticando continuamente con gli uomini alla fine si debba immascolinar troppo; prevedere che la casa, senza più le cure assidue, intelligenti, amorose della donna debba perdere quella poesia intima e cara, che è la maggiore attrattiva del matrimonio per l'uomo; supporre che la donna, cooperando anch'essa col proprio guadagno al mantenimento della casa, non debba aver più per l'uomo quella devozione e quel rispetto, di cui tanto essa si compiace: non sono pregiudizi; sono tristi necessità per cui la composizione ideale del feminismo si scompone e si scioglie nella questione più vasta delle tristissime condizioni economiche $\mathrm{e}$ sociali dei giorni nostri. Si scioglie, senza lasciar residui, signor mio, creda pure. Soltanto, quel po' di pellacchia sgonfiata [...] .

- Vorrei - io dissi piano, in un orecchio al dottore, prima d'alzarmi per tor commiato - vorrei ora sentir come la vede la signorina sua figliuola [. . . ] .

- Caro signore - mi rispose aprendo le braccia, il dottor Paulo Post. - Le donne non possono veder da lontano questa questione. O potrebbero a un solo patto: che avessero cioè il marito vicino, mi spiego?

Io scappo ancora. (1071-72)

Le parole apparentemente "sensate" del padre il quale però impedisce alla figlia di dire la sua, la laconicità del narratore che "scappa" senza insistere e senza ribattere, lasciano incerti se si tratti di anti-femminismo, di una satira dell'antifemminismo, o di un'antifemminismo "mascherato" da satira. ${ }^{12}$

Illuminante invece è il confronto con la novella Pari, composta nel $1907 \mathrm{ma}$ pubblicata soltanto nel 1911. Qui, usando il discorso indiretto libero, il narratore attribuiva a due scapoli "gemelli" $i$ cui rapporti con le donne possono dirsi per lo meno problematici lo stesso identico ragionamento, parola per parola, che avrebbe poi messo direttamente in bocca al dottore Paulo Post (non cito i pezzi uguali):

Bartolo Barbi e Guido Pagliocco, entrati insieme per concorso al Ministero dei Lavori Pubblici da vice-segretarii [. . .] erano divenuti, dopo tanti anni di vita comune, indivisibili amici.

Abitavano insieme, in due camere ammobiliate al Babuino. Per grazia particolare della vecchia padrona di casa, che si lodava tanto di loro, avevano anche il salottino a disposizione, ove solevano passar le serate, quando - sempre d'accordo stabilivano di non andare a teatro o a qualche caffe-concerto. Giocavano a dadi o a scacchi o a dama, intramezzando alle partite pacate e sennate conversazioncine $o$ sui superiori o sui compagni d'ufficio o su le questioni politiche del momento o anche su le arti belle, di cui si reputavano con una certa soddisfazione estimatori non volgari [...].

Nelle loro conversazioni serali, Barbi e Pagliocco avevano definito il feminismo questione essenzialmente economica. (413)

E chiaro che qui il ragionamento ha la funzione di razionalizzare o mascherare paure ancestrali rimosse e riportate a galla dalle rivendicazioni del femminismo 
che hanno scomposto il vecchio assetto sociale. Il desiderio femminile acquista risvolti nuovi - desiderio di lavoro, di guadagno, di diritti legali, di mezzi espressivi - proprio nel momento in cui le energie maschili non sanno più con certezza dove dirigersi. Ciò non fa che mettere a nudo - non a caso la raccolta in cui è inserito il racconto s'intitola $L a$ vita nuda - l'inettitudine maschile che si nasconde male dietro l'attacco al femminismo e il rifiuto di sposarsi:

Avevano da un pezzo non solo chiuso la porta del cuore alla donna, ci avevano anche messo il catenaccio. Non ne aspettavano più, neanche in sogno. Che se talvolta qualche desiderio monello saltava dentro all'improvviso per la finestra degli occhi, subito la ragione arcigna lo cacciava via a pedate [. . .] .

Mai e poi mai Barbi e Pagliocco avrebbero sposato una donna emancipata, impiegata, padrona di sé. Non perché volessero schiava la moglie, ma perché tenevano alla loro dignità maschile e non avrebbero saputo tollerare che questa, di fronte ai guadagni della moglie, restasse anche minimamente diminuita. Metter su casa, d'altra parte, con lo scarso stipendio da segretario, sarebbe stata una vera e propria pazzia, e dunque niente; non ci pensavano nemmeno. (413-14)

Senonché, i casi della vita fanno sì che un giomo i due scapoli si trovino a venir meno alle loro risoluzioni. Vittime dell'intraprendenza della moglie del capo-ufficio, la quale si diverte ad accoppiare gli impiegati scapoli del marito con le figlie nubili delle signore amiche, si convincono a sposare due "fanciulle sagge e modeste, econome e massaje" (412-13):

Le signorine Gandini e Monti avevano, per altro, una discreta doticina; erano poi tra loro da tanti anni amiche inseparabili, e non avrebbero perciò né sciolto, né allentato d'un punto il legame che anch'essi uniti; e dunque, giudiziosamente, al solito, $\mathrm{i}$ due amici stabilirono di prendere a pigione due appartamenti contigui, per seguitare a vivere insieme, uniti e separati a un tempo. (415)

Unico impiccio è il fatto che una delle ragazze ha una dote inferiore a quella dell'altra. Il Pagliocco s'impunta, perché vuole che tutto sia "pari". E allora, con qualche sacrificio la madre della ragazza meno "dotata" riesce a racimolare i soldi necessari per mettere le due spose "alla pari": "Pari in tutto, anche le doti dovevano essere pari" (415). Il finale del racconto vede $i$ due amici anche parimenti traditi dalle mogli che si sono invaghite ciascuna del fratello dell'altro, con la giustificazione che, siccome $\mathrm{i}$ due giovani somigliavano molto ai fratelli più grandi, e siccome i due mariti "quasi formavano un uomo solo da amare insieme" (415-16) le mogli non facevano altro che "completare" l'uomo che avevano già davanti col fratello dell'altro!

La novella Pari permette quindi di collocare l'atteggiamento pieno di "buon senso" del dottor Paulo Post di Feminismo in una nuova luce e spiega l'ambiguità dell'articolista. A parte il fatto che ciascuno dei "gemelli" viene caratterizzato molto efficacemente come "metà-uomo" che quindi non riesce a soddisfare la moglie, il racconto, con tutto ciò che ha di geometrico, di paradossale e di assurdo, istituisce un chiaro rapporto di causa ed effetto fra paura 
dell'alterità femminile e anti-femminismo, fra narcisismo patologico e desiderio di "solitudine maschile": quella che vivono Barbi e Pagliocco prima del matrimonio, quella che difende con tanto accanimento Liolà e, per tornare al nostro punto di partenza, quella in cui va a finire Mattia Pascal.

Il desiderio di Mattia Pascal appare quindi il desiderio di tanti maschi del mondo pirandelliano: essere soli e semmai condividere la solitudine con qualche amico-gemello e vivere filosofando, tenendo così a bada le proprie paure e riempendo il vuoto che viene a crearsi in un'esistenza così concepita. Il che equivale a dire che il suo desiderio "vero", in quanto maschio, è quello di morire. Ed è ciò che fa ben tre volte, simbolicamente: prima a Miragno, approfittando dell'identificazione sbagliata del cadavere nella Stía per fuggire dalla moglie, poi a Roma, simulando il suicidio di Adriano Meis sul Lungotevere per fuggire da Adriana, e finalmente, di ritorno a Miragno, rifiutando di correggere la sua situazione anagrafica per sottrarsi ai suoi doveri coniugali e poter vivere come il fu Mattia Pascal.

McGill University

\section{NOTE}

1 Per una discussione delle interpretazioni critiche del romanzo si veda Stocchi-Perucchio 123; si veda anche la bibliografia (147-56).

2 Per una discussione della questione in relazione al Fu Mattia Pascal si veda Marchese.

3 Angelo Marchese parla della "compresenza di due modelli diversi di narrazione, non del tutto perfettamente integrati: l'uno, avventuroso, tende a dilatare la fabula in forma di intrigo; l'altro, psicologico-esistenziale, è contratto sulla causalità interiore del personaggiovoce narrante" (542).

4 Sulla donna come oggetto di scambio nelle società patriarcali si veda lrigaray: il saggio apparve per la prima volta in Sessualità e politica (Milano: Feltrinelli, 1978). Anche Rubin, trad. it. "Lo scambio delle donne: una rilettura di Marx, Engels, Lévi-Strauss e Freud", Nuova DWF 1 (1976): 23 sgg. Per una discussione di lrigaray e di Rubin, e una prospettiva che fonde la lettura di Lévi-Strauss e di Marx fatta da Rubin con spunti derivati dalle teorie di René Girard, si veda Sedgwick. Su questo tema in Pirandello si veda il capitolo di Gunsberg; Spizzo; e Gardair.

5 Gaspare Giudice (313) cita una lettera del 1916 in cui Pirandello scrive al figlio a proposito di Liolà: ". . . L'ho scritta in quindici giomi . . . ; ed è stata la mia villeggiatura. Di fatti si svolge in campagna. Mi pare d'averti già detto che il protagonista è un contadino-poeta, ebbro di sole. Ė così gioconda che non pare opera mia ...."

6 Anche Marchese parla di una psicologia sociale, ma la intende in termini leggermente diversi: “. . . l'etichetta piccolo-borghese non ha, per noi, una referenza esclusivamente sociologica, se è vero che il codice storico-politico non è né rilevante, né decisivo nell'ermeneutica del testo, ma serve piuttosto a individuare una psicologia socialmente determinata (ad esempio, l'atteggiamento nei riguardi delle relazioni amorose inconcepibili fuori del matrmonio, il mito della famiglia come 'nido'. . .), dietro alle quali si agitano $\mathrm{i}$ fantasmi interiori e inconsci di un io diviso" (543).

7 Luperini osserva giustamente che Pirandello trasforma "i protagonisti delle sue narrazioni in astrazioni personificate attraverso un duplice movimento: togliendo loro concretezza vitale mediante la loro trasformazione in personaggi, e rendendoli 'generici' mediante la loro 
trasformazione in figure o emblemi di un discorso esclusivamente intellettuale e di una ragione puntogliosamente argomentante" (221).

8 Marchese 543; Luperini parla di "una radicale desoggettivazione dell'interiorita" (249).

9 Mattia non ha conosciuto il padre, Serafino Gubbio non vi accenna nemmeno, Vitangelo Moscarda ne parla trattandolo semplicemente da "usuraio", il Padre nei Sei personaggi sfiora l'incesto, i padri di Rocco e Marta nell'Esclusa sono grottesche caricature prepotenti ed autoritarie, Giustino Boggiolo, il marito di Silvia Roncella in Suo marito ha la madre ma non il padre, come anche l'inetto Pepè Alletto del lungo racconto ll turno.

10 Il romanzo Suo marito è la storia di una scrittrice che diventa "merce di scambio" per il marito imprenditore, costringendola finalmente ad abbandonarlo. (Rinvio ad un altro mio saggio, "La scrittrice"). Nel romanzo Quaderni di Serafino Gubbio operatore, la figura di Varia Nestoroff, la donna "fatale" di tanta letteratura e cinema del suo tempo, dopo essere stata passata da Giorgio Mirelli ad Aldo Nuti viene da Serafino recuperata ad una sua dimensione soggettiva, per essere poi freddata da un colpo di pistola dal Nuti imbestialito. II parallello che Pirandello istituisce fra la Nestoroff e la tigre catturata dall'uomo e ridotta ad oggetto costretto a vivere in una gabbia secondo le regole dell'uomo non mi pare mi abbia bisogno di ulteriori elucidazioni. Anche La nuova colonia è la storia di una donna ridotta ad oggetto di scambio dal marito che l'abbandona nel momento in cui ha la possibilita di aumentare il suo prestigio personale attraverso il matrimonio con la figlia di un uomo potente. Improvvisamente un terremoto distrugge l'isola in cui vivono, lasciando in vita solo la Spera e il figlio, come se la natura volesse vendicarsi dell'oltraggio fattale dal marito e da tutta una societa basata sull'oggettivazione delle donne.

11 L'articolo ora è reperibile in Saggi, poesie, scritti vari.

12 Il libro di Gunsberg contiene un'analisi dettagliata dell'articolo (195-207) e conclude che si tratta di anti-femminismo; non tiene presente, perd, la novella Pari.

\section{OPERE CITATE}

Alonge, Roberto. Pirandello tra realismo e mistificazione . Napoli: Guida, 1972.

"Madri, puttane, schiave sessuali e uomini soli". Studi pirandelliani. Dal testo al sottosuolo. Ed. Roberto Alonge. Bologna: Pitagora, 1986. 91-110.

Gardair, Jean-Michel. Pirandello. Fantasmes et logique du double. Paris: Larousse, 1972.

Girard, René. Mensonge romantique et vérité romanesque. Paris: Grasset, 1961.

Giudice, Gaspare. Pirandello. Torino: UTET, 1973.

Gunsberg, Maggie. "Camaraderie in Competition: Male Bonding and Male Rivalry". Patriarchal Representations. Gender and Discourse in Pirandello's Theatre. Oxford/Providence: Berg, 1994. 64-99.

Irigaray, Luce. "Le marché des femmes". Ce sexe qui n'en est pas un. Paris: Les Editions de Minuit, 1977. 165-85.

Kroha, Lucienne. "La scrittrice come oggetto di scambio nel romanzo Suo marilo di Pirandello". Les femmes-écrivains en lialie (1870-1920), ordres et liberlés. Ed. Emmanuelle Genevois. Chroniques italiennes (Université de la Sorbonne Nouvelle) 39/40 (1994): 223-42.

Leone De Castris, Arcangelo. Storia di Pirandello. Bari: Laterza, 1971.

Lugnani, Lucio. "L'infanzia felice". L'infanzia felice e altri saggi su Pirandello. Napoli: Liguori, 1986. 102-65.

Luperini, Romano. L'allegoria del moderno. Roma: Editori Riuniti, 1990.

Marchese, Angelo. "I codici nascosti del Fu Mattia Pascal". Humanitas 42.4 (1987): 526-48

Masiello, Vitilio. "La mosca nella bottiglia. Introduzione alla lettura del Fu Matria Pascal". Lo strappo nel cielo di carta. Introduzione alla lettura del Fu Matria Pascal. Premessa di Enzo Lauretta. Roma: La Nuova Italia Scientifica, 1988. 67-82.

Mazzacurati, Giancarlo. Pirandello nel romanzo europeo. Bologna: Il Mulino, 1987.

Pirandello, Luigi. Il fu Mattia Pascal. Milano: Mondadori, 1971. 
"Feminismo". Saggi, poesie, scritti vari. Ed. Manlio Lo Vecchio-Musti. II ed. Milano: Mondadori, 1965. 1068-72.

Pari. Novelle per un anno. Vol. 1. VII ed. Milano: Mondadori, 1969. 409-17.

Rosowsky, Giuditta. "Lo schema narrativo dell' autobiografia nel Fu Mattia Pascal". Il romanzo di Pirandello. Ed. Enzo Lauretta. Palermo: Palumbo, 1976. 77-93.

Rubin, Gayle. "The Traffic in Women: Notes Toward a Political Economy of Sex". Toward an Anthropology of Women. Ed. Rayna Reiter. New York: Monthly Review Press, 1975. 157-210.

Sedgwick, Eve Kosofsky. "Gender Assymetry and Erotic Triangles". Between Men. English Literature and Male Homosocial Desire. New York: Columbia UP, 1985. 21-27.

Spizzo, Jean. "Pirandello: Théatre du reflet, théatre du conflit". A. Bouissy et al., Lectures pirandelliennes. Paris: Centre de recherche de l'Université de Paris VIII - Vincennes, 1978. 218-22.

Stocchi-Perucchio, Donatella. Pirandello and the Vagaries of Knowledge. A Reading of Il fu Mattia Pascal. Saratoga, Calif.: ANMA Libri, 1991. 